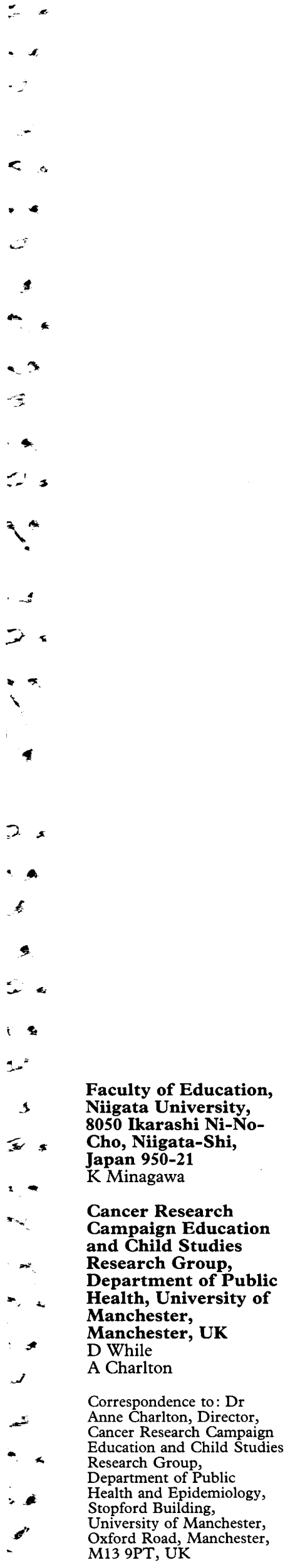

\title{
Smoking and self-perception in secondary school students
}

\author{
Koh-ei Minagawa, David While, Anne Charlton
}

\begin{abstract}
Objective - To carry out a cross-sectional pilot baseline survey to investigate possible links between teenage smoking and self-perception in six domains by means of a validated psychological measure.
\end{abstract} Design-Anonymous self-administered questionnaires including the Harter SelfPerception Profile for Children (1985). Subjects-Eight hundred and thirty students ( 407 boys and 423 girls) aged 11 to 15 years in two state secondary schools in Northern England.

Main outcome measures-Chi-squared tests, odds ratios, and one-way analysis of variance for age trends with regard to self-perception and smoking. Correlation between domains, and regression analysis with domains and with smoking as the dependent variable.

Results - The girls' self-perception was significantly lower than that of the boys for scholastic, athletic, physical appearance, and global self-worth domains. Girls' self-perception scores fell significantly with increasing age as follows: scholastic $(p=0.0300)$, physical appearance $(p<0.0001)$, behavioural $(p<0.0001)$ and global self-worth $(p<0.0001)$ domains, whilst boys showed no significant changes. Smoking was significantly linked to low self-perception scores in girls in scholastic competence $(p<$ $0.0001)$, physical appearance $(p=0.0011)$, behavioural conduct $(p<0.0001)$, and global self-worth domains $(p<0.0001)$. For boys, only in scholastic $(p=0.0010)$ and behavioural ( $p<0.0001)$ self-perception was smoking linked with low scores. Higher self-perception scores in the social competence domain were associated with smoking in boys and girls, but not significantly. Associations were found between most of the six domains, the most significant being between physical appearance and global self-worth for girls, and the only pair that showed no association for either boys or girls was behavioural conduct and social acceptance. Logistic regression analysis showed a combination of friends' smoking, school attended, and self-perception with regard to behaviour and social acceptance to be the most significant factors associated with smoking in girls, with male family members' smoking being significant for boys rather than school or social acceptance.

Outcome-A longitudinal study is to be conducted to investigate possible causality. The meaning of the findings for the health-promoting school are discussed.

(Tobacco Control 1993; 2: 215-21)

\section{Introduction}

A major cause of concern in recent years is the high prevalence of smoking among teenage girls and young women. Although smoking prevalence in Great Britain has been decreasing steadily over the past 20 years, it has fallen more slowly in women than in men, and in the past few years has actually risen in younger females. For example, prevalence of cigarette smoking rose from $28 \%$ in 1988 to $32 \%$ in 1990 among 16- to 19-year-old women and from $37 \%$ to $39 \%$ in 20 - to 24 -year-old women over the same period. ${ }^{1}$

National surveys of secondary school pupils since 1982 have shown that, whilst girls experiment with smoking slightly later than boys do, their prevalence of regular smoking overtakes that of boys at about the age of 13 years. ${ }^{2}$ This pattern is consistent throughout most of the industrialised world. ${ }^{3}$ Therefore, it appears that the increased uptake of smoking by girls is grounded in the mid-teen years and the increases observed in young women are probably due largely to a cohort effect rather than new recruitment to smoking in these older age groups.

A considerable amount is known about which young women are at most risk. ${ }^{4}$ For example, post-school factors include having lower socio-economic status, being a lone parent, having high stress factors, and having a poor internal locus of control which leads to perceived lack of personal competence in affecting the progress or outcome of life events. ${ }^{5}$ The foundations of many of these later conditions could be laid at school and, all too often, smoking behaviour begins at the same time. The very addictive nature of smoking soon becomes established, and these young women have then added smoking to their other disadvantages. ${ }^{6}$ What is not yet clear is when and how psychological factors in girls differ from those of boys with regard to onset or maintenance of smoking.

The cross-sectional pilot study described in this paper was carried out in order to provide 
a baseline to investigate this process in more detail.

\section{Sample}

The two schools in this study were selected because $85.9 \%$ of students aged 11 to 15 (Years 7 to 11 ) attend this type of secondary school. ${ }^{7}$ The total number of students in the two schools represented $5 \%$ of students in the age range in that region of northern England. The schools were selected by allocating numbers to all the schools and drawing the names of the participating ones by means of a table of random numbers. The age and gender distribution reflects that of this age range in England at present. These two schools formed part of the sample for a larger study. The 11-15 age range was selected for separate discussion in this paper because it is the range of British national surveys and has been found to be particularly critical in the onset of smoking.

Responses were received from all the 1073 students in the age range who were present in the two schools at the time the questionnaire was administered. The absentee rate on that date was approximately $13 \%$. Of the 1073 questionnaires 41 were rejected from the analyses due to inconsistencies between the check questions and their reported smoking status or failure to complete the smoking question. Using the chi-squared test, it was found at the $1 \%$ level that this sub-sample of 41 students did not differ significantly from the remaining 1032 students with regard to the school they attended, gender, age, or smoking status of their mother, father, brothers, sisters, or best friends. At the $5 \%$ level, the subsample of 41 students contained significantly fewer students who stated that their best friends did not smoke $\left(\chi^{2}=7.49 ; \mathrm{df}=2 ; \mathrm{p}=\right.$ $0.024)$; however no other characteristics were significantly different from the remaining students at this level.

Of these 1032 respondents, a further 202 questionnaires had to be rejected due to incomplete responses to the 36 self-perception questions. After stratifying for gender, it was found at the $1 \%$ level that this sub-sample of 202 students did not differ significantly from the remaining 830 respondents with regard to their own smoking status; the smoking status of parents, siblings, and best friend; school attended; or age. However, at the $5 \%$ level, a significant difference was identified with regard to completion of the Harter self-perception questions by boys. Fifteen-year-old boys were more likely than 11 - to 14 -year-olds to respond $\left(\chi^{2}=11.18 ; \mathrm{df}=4 ; \mathrm{p}=0.025\right)$. In all, 830 questionnaires went into the analysis ( 407 boys and 423 girls).

\section{The Questionnaire}

Three elements were contained in the questionnaire as follows:

(a) Prevalence questions relating to the respondents themselves, their families, and their friends. The smoking prevalence question used was the standard six-category one developed by Bewley ${ }^{8}$ and used for most surveys of children's smoking prevalence. In this age group a regular smoker is defined as a young person who smokes at least one cigarette a week.

(b) Questions on refusal skills and methods to identify effective ways of resisting peer pressure.

(c) Self-perception questions, using Harter's Self-Perception Profile for Children questionnaire, ${ }^{9}$ adapted for use with British children. This 36-question instrument measures self-perception in five domains and also global self-worth. The specific domains are scholastic competence, social acceptance, athletic competence, physical appearance, and behavioural conduct. The only major change made to the questionnaire was the replacement throughout of the word "kids" by "people". The questionnaire for children rather than the more complex one for adolescents was used in this instance because younger children were included in the wider survey of which this study forms a part and comparability could not otherwise have been achieved.

The Harter Self-Perception Profile for Children is a widely used and validated instrument for psychological measurement in children. It is one of the most acceptable means of assessing self-perception standards, and is used by child psychologists and in a wide variety of studies with a psychological basis. As far as the authors are aware, this measure has been used once previously in tobacco-related behaviour studies in the $\mathrm{UK}^{10}$ and its value in such studies is clear.

Only the self-perception aspects of the survey are discussed in this paper.

\section{Methodology}

The questionnaires were mailed to the schools in time to be administered to all the students who were present on a single day during the week of 13-17 January 1992. All the pupils in each school completed the questionnaire simultaneously in class groups supervised by their class teachers. When they had finished, each pupil sealed his or her anonymous questionnaire into a plain envelope. The sealed envelopes in class bundles were then mailed back to the researcher.

It would have been useful to validate the responses with saliva cotinine tests, but with such large numbers, the cost would have been prohibitive even for a sample test. In fact, studies have shown that very few, perhaps about $1 \%$ of children, do lie about their smoking status, ${ }^{2}$ but nevertheless as much as possible was done to ensure accuracy. A crosscheck question which was used in surveys ${ }^{2}$ of smoking among secondary school children conducted by the Office of Population Censuses and Surveys was included, as was a question in which the respondents stated how many cigarettes they had smoked in the previous day or week. Appropriate reclassification or rejection of questionnaires was made 


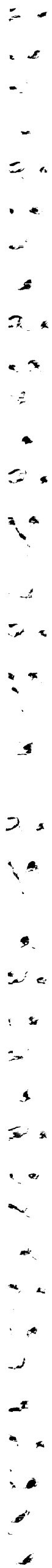

when these responses did not agree with the response to the standard smoking category question. The categories are: (1) I have never smoked a cigarette; (2) I have only ever tried smoking once; (3) I used to smoke sometimes, but I don't now; (4) I smoke sometimes, but I don't smoke as much as one cigarette a week; (5) I usually smoke between one and six cigarettes a week; (6) I usually smoke more than six cigarettes a week. For purposes of analysis these categories were sometimes collapsed to three, ie, never smoked (1); sometime smoker $(2,3$, and 4$)$; regular smoker 5 and 6$)$. When two categories were used they were: not current smoker (1,2, and 3) and current smoker $(4,5$, and 6$)$.

Analysis was carried out by SPSSX, and a number of different statistical procedures were used to calculate the following:

- chi-squared tests and odds ratios for simple relationships

- one-way analysis of variance for age trends

- Pearson correlation coefficient for correlations between domains with regard to age and gender

- forward stepwise multiple linear regression with main affects and first-order interactions with the domains as separate outcome variables

- forward stepwise logistic regression, in which all the variables (ie, school attended, age, gender, mother's smoking, father's smoking, brothers' and sisters' smoking, best friend's smoking) and the six domains of self-perception were entered into the model with current smoking as the dependent variable. First order interactions between the significant variables were also investigated

- cluster analysis was considered, but problems arose due to the terminology used by the respondents to describe their class.

The same school class could be variously named by year only, by a number and letter code, the teacher's name, or the room number. The confusion was further compounded by the existence of cross-class streaming for some subjects. The initial reaction, when age/year groups were examined, was that it would be well worthwhile developing a method in the future for precoding a class reference before distributing questionnaires to the students.

\section{Results}

PREVALENCE OF SMOKING

The prevalence of regular smoking (ie, at least one cigarette per week) is shown in table 1.
Table 1 Prevalence of regular smoking of at least one cigarette per week $(\%(n / b a s e))$

\begin{tabular}{lccccc}
\hline & \multicolumn{5}{c}{ Age (years) } \\
\cline { 2 - 6 } & 11 & 12 & 13 & 14 & 15 \\
\hline Boys & $0(0 / 46)$ & $1(1 / 95)$ & $5(5 / 94)$ & $4(3 / 79)$ & $12(11 / 93)$ \\
Girls & $0(0 / 54)$ & $0(0 / 89)$ & $10(10 / 101)$ & $14(11 / 81)$ & $26(25 / 98)$
\end{tabular}

The sudden increase in prevalence among girls between the ages of 13 and 14 is very apparent, as is the fact that smoking prevalence then remained significantly higher among girls than among boys up to the age of 15 .

\section{SELF-PERCEPTION}

By stratifying the sample with respect to age and gender and examining the distribution of each of the six domains within each stratum, it was established that at the $1 \%$ level there were no violations of normality. Using one-way analysis of variance it is shown in table 2 that the girls' self-perception, with regard to scholastic achievement, physical appearance, behavioural conduct, and global self-worth fell significantly with increasing age between 11 and 15 years. There were no parallel decreases among the boys, whose self-perception in almost all domains at every age was generally higher than that of the girls. It must be borne in mind that this is a cross-sectional sample with the restrictions on interpretation which such a study imposes. When the results of the longitudinal study currently in progress are available, it will be possible to determine cohort effects.

\section{SELF-PERCEPTION AND SMOKING}

The relationship between self-perception and smoking was complex but nevertheless a clear pattern emerged. Analysis of variance and multiple linear regression modelling were used to clarify the relationships, and odds ratios (ORs) were calculated using the "never smoked" category as the baseline.

(a) Scholastic competence, as perceived by the respondents, was inversely associated with increased risk of being a smoker. Using one-way analysis of variance table 3 shows that this association was highly statistically significant for both boys $(p=0.0010)$ and girls $(\mathrm{p}<0.0001)$. It has been known that underachievers are more likely than "high fliers" to be smokers, but the self-perception score used here was not a measure of actual scholastic achievement, but the

Table 2 Mean self-perception scores in male $(M)$ and female $(F)$ students (Harter Self-Perception Scale)

\begin{tabular}{|c|c|c|c|c|c|c|c|c|c|c|c|c|}
\hline \multirow[b]{2}{*}{ Age (years) } & \multicolumn{2}{|c|}{$\begin{array}{l}\text { Scholastic } \\
\text { competence }\end{array}$} & \multicolumn{2}{|c|}{$\begin{array}{c}\text { Social } \\
\text { acceptance }\end{array}$} & \multicolumn{2}{|c|}{$\begin{array}{c}\text { Athletic } \\
\text { competence }\end{array}$} & \multicolumn{2}{|c|}{$\begin{array}{l}\text { Physical } \\
\text { appearance }\end{array}$} & \multicolumn{2}{|c|}{$\begin{array}{l}\text { Behavioural } \\
\text { conduct }\end{array}$} & \multicolumn{2}{|c|}{$\begin{array}{c}\text { Global } \\
\text { self-worth }\end{array}$} \\
\hline & $M$ & $F$ & $M$ & $F$ & $M$ & $F$ & $M$ & $F$ & $M$ & $F$ & $M$ & $F$ \\
\hline $\begin{array}{l}11 \\
12 \\
13 \\
14 \\
15\end{array}$ & $\begin{array}{l}2.68 \\
2.82 \\
2.72 \\
2.80 \\
2.71\end{array}$ & $\begin{array}{l}2.80 \\
2.73 \\
2.63 \\
2.59 \\
2.57\end{array}$ & $\begin{array}{l}2.97 \\
3.06 \\
3.06 \\
2.97 \\
3.03\end{array}$ & $\begin{array}{l}2.93 \\
3.07 \\
3.00 \\
2.94 \\
2.92\end{array}$ & $\begin{array}{l}2.97 \\
3.04 \\
2.92 \\
2.85 \\
2.86\end{array}$ & $\begin{array}{l}2.62 \\
2.60 \\
2.40 \\
2.50 \\
2.42\end{array}$ & $\begin{array}{l}2.91 \\
2.87 \\
2.78 \\
2.72 \\
2.77\end{array}$ & $\begin{array}{l}2.53 \\
2.48 \\
2.24 \\
2.20 \\
2.18\end{array}$ & $\begin{array}{l}2.81 \\
2.85 \\
2.73 \\
2.73 \\
2.69\end{array}$ & $\begin{array}{l}3.06 \\
2.95 \\
2.75 \\
2.67 \\
2.70\end{array}$ & $\begin{array}{l}3.13 \\
3.15 \\
3.07 \\
3.05 \\
3.08\end{array}$ & $\begin{array}{l}3.01 \\
3.01 \\
2.83 \\
2.75 \\
2.64\end{array}$ \\
\hline $\begin{array}{l}\text { One way analysis } \\
\text { of variance } p=\end{array}$ & 0.4276 & 0.0300 & 0.7333 & 0.3607 & 0.2395 & 0.1350 & 0.2683 & $<0.0001$ & 0.2798 & $<0.0001$ & 0.6561 & $<0.0001$ \\
\hline
\end{tabular}


Table 3 Mean self-perception scores related to self-reported smoking status in males $(M)$ and females $(F)$

\begin{tabular}{|c|c|c|c|c|c|c|c|c|c|c|c|c|}
\hline \multirow{2}{*}{$\begin{array}{l}\text { Smoking } \\
\text { status }\end{array}$} & \multicolumn{2}{|c|}{$\begin{array}{l}\text { Scholastic } \\
\text { competence }\end{array}$} & \multicolumn{2}{|c|}{$\begin{array}{c}\text { Social } \\
\text { acceptance }\end{array}$} & \multicolumn{2}{|c|}{$\begin{array}{c}\text { Athletic } \\
\text { competence }\end{array}$} & \multicolumn{2}{|c|}{$\begin{array}{c}\text { Physical } \\
\text { appearance }\end{array}$} & \multicolumn{2}{|c|}{$\begin{array}{l}\text { Behavioural } \\
\text { conduct }\end{array}$} & \multicolumn{2}{|c|}{$\begin{array}{l}\text { Global } \\
\text { self-worth }\end{array}$} \\
\hline & $M$ & $F$ & $M$ & $F$ & $M$ & $F$ & $M$ & $F$ & $M$ & $F$ & $M$ & $F$ \\
\hline Never smoked & 2.83 & 2.77 & 3.00 & 2.94 & 2.93 & 2.52 & 2.82 & 2.40 & 2.89 & 3.02 & 3.13 & 2.93 \\
\hline $\begin{array}{l}\text { Smoked at } \\
\text { some time }\end{array}$ & 2.63 & 2.53 & 3.04 & 3.00 & 2.92 & 2.51 & 2.77 & 2.20 & 2.54 & 2.63 & 3.01 & 2.74 \\
\hline Regular smoker & 2.57 & 2.37 & 3.28 & 3.11 & 2.98 & 2.34 & 2.83 & 2.12 & 2.40 & 2.18 & 3.16 & 2.54 \\
\hline $\begin{array}{c}\text { One-way analysis } \\
\text { of variance } p=\end{array}$ & 0.0010 & $<0.0001$ & 0.0633 & 0.1155 & 0.9387 & 0.2533 & 0.7575 & 0.0011 & $<0.0001$ & $<0.0001$ & 0.1166 & $<0.0001$ \\
\hline
\end{tabular}

way in which each student perceived himor herself to be achieving. The two are not necessarily identical. A low self-perception score could reflect a rejection of this value instead of, or as well as, an expression of personal achievement. Girls who had a score below the mean for their age group in this domain had a higher risk of being regular smokers $(\mathrm{OR}=3.3 ; 95 \%$ confidence interval $(\mathrm{CI})=1.7-6.3)$ and of being sometime smokers $(\mathrm{OR}=2.7 ; 95 \% \mathrm{CI}=$ $1.7-4.1)$, whereas boys had a risk of being sometime smokers $(\mathrm{OR}=1.6 ; 95 \% \mathrm{CI}=$ 1.04-2.5).

(b) Social acceptance was the only domain in which smokers were most likely to have the highest scores. Again this applied to boys as well as to girls, although it was not statistically significant (boys, $\mathrm{p}=0.0633$; girls, $p=0.1155)$. However, boys with selfperception scores in the social domain above the mean were more likely to be sometime smokers $(\mathrm{OR}=6.8 ; 95 \% \mathrm{CI}=$ 1.9-23.8).

(c) Athletic competence was not significantly related to smoking status (girls, $\mathrm{p}=0.2533$; boys, $\mathrm{p}=0.9387$ ). This finding may surprise some people in that an approach often advocated for smoking intervention is to raise young people's athletic perception.

(d) Physical appearance was the domain in which there was the greatest variation in its association with smoking between boys and girls (girls, $\mathrm{p}=0.0011$; boys, $\mathrm{p}=$ 0.7575). Among the girls, self-perception in this domain fell steeply from neversmokers through sometime smokers to regular smokers. Girls who scored below the mean in this domain were more likely to be sometime or regular smokers $(\mathrm{OR}=$

Table 4 Pearson correlation coefficients between domains of self-perception (by gender)

\begin{tabular}{llllll}
\hline & $\begin{array}{c}\text { Scholastic } \\
\text { competence }\end{array}$ & $\begin{array}{c}\text { Social } \\
\text { acceptance }\end{array}$ & $\begin{array}{c}\text { Physical } \\
\text { appearance }\end{array}$ & $\begin{array}{c}\text { Athletic } \\
\text { competence }\end{array}$ & $\begin{array}{c}\text { Behavioural } \\
\text { conduct }\end{array}$ \\
\hline Boys & & & & & \\
Social acceptance & $0.1275^{\star}$ & & & & \\
Physical appearance & $0.2713^{\star \star}$ & $0.3273^{\star \star}$ & & & \\
Athletic competence & $0.1460^{\star \star}$ & $0.34888^{\star \star}$ & $0.2900^{\star \star}$ & & \\
Behavioural conduct & $0.4849^{\star \star}$ & 0.0389 & $0.2174^{\star \star}$ & -0.0219 & \\
Global self-worth & $0.4498^{\star \star}$ & $0.3805^{\star \star}$ & $0.5772^{\star \star}$ & $0.1986^{\star \star}$ & $0.4750^{\star \star}$ \\
Girls & & & & & \\
Social acceptance & $0.1622^{\star \star}$ & & & & \\
Physical appearance & $0.3344^{\star \star}$ & $0.2161^{\star \star}$ & & & \\
Athletic competence & $0.2141^{\star \star}$ & $0.3568^{\star \star}$ & $0.3852^{\star \star}$ & & \\
Behavioural conduct & $0.4503^{\star \star}$ & 0.0531 & $0.2952^{\star \star}$ & $0.1316^{\star \star}$ & \\
Global self-worth & $0.4524^{\star \star}$ & $0.4165^{\star \star}$ & $0.5961^{\star \star}$ & $0.3792^{\star \star}$ & $0.4275^{\star \star}$ \\
\hline
\end{tabular}

* significant $\mathrm{p}<0.05 ;{ }^{\star \star}$ significant $\mathrm{p}<0.01$ (2-tailed).
$1.7 ; 95 \% \quad \mathrm{CI}=1.4-2.7$ and $\mathrm{OR}=2.2$ $95 \% \mathrm{CI}=1.2-4.2$ ). In boys, who had much higher self-perception than girls, there was virtually no difference whatever related to their smoking status.

(e) Behavioural conduct was predictably related to smoking in both boys $(\mathrm{p}<0.0001)$ and girls $(\mathrm{p}<0.0001)$. Those who see themselves as "good" are least likely to be smokers. Other questions also showed that these students with higher scores for behavioural conduct had a wider range of refusal skills when offered cigarettes. The risk for girls with self-perception scores below the mean being regular smokers (OR $=44.7 ; 95 \% \mathrm{CI}=13.4-149.4)$ was greater than for boys $(\mathrm{OR}=7.0 ; 95 \% \mathrm{CI}=$ 2.3-21.7). For girls who scored below the mean there was also a significant risk of being a sometime smoker $(\mathrm{OR}=4.7 ; 95 \%$ $\mathrm{CI}=3.0-7.5$ ), and similarly for boys (OR $=2.9 ; 95 \% \mathrm{CI}=1.9-4.5$ ).

(f) Global self-worth was another domain in which boys scored much higher than girls, but only in girls was it significantly related to smoking status (boys, $\mathrm{p}=0.1166$; girls, $\mathrm{p}<0.0001$ ). Global self-worth below the mean was associated with regular smoking in girls $(\mathrm{OR}=3.2 ; 95 \% \mathrm{CI}=1.7-6.2)$ but not in boys, and also with sometime smoking in girls $(\mathrm{OR}=1.8 ; 95 \% \mathrm{CI}=$ 1.2-2.8) but not in boys.

CORRELATIONS BETWEEN DOMAINS OF SELFPERCEPTION

Of course, the picture is not as simplistic as the above results might suggest. The various domains are interrelated and Pearson's Correlation Co-efficients were calculated for boys and girls separately. As table 4 shows, significant correlations were found between most of the domains for both genders. The largest significant association was between physical appearance and global self-worth in girls. The domains between which no significant correlations were found for either boys or girls were behavioural conduct and social acceptance. Correlations within age groups were calculated but their complexity was such that they could better be used to provide the substance of another paper.

BACKGROUND FACTORS RELATED TO DOMAINS OF SELF-PERCEPTION

In view of the complex pattern presented by the social background factors, self-perception 
Table 5(a) Boys: multiple linear regression models (parameter estimate, $(S E)$ ) with interactions with domains as the dependent variables $(\star=N S)$

\begin{tabular}{|c|c|c|c|c|c|c|}
\hline & $\begin{array}{l}\text { Scholastic } \\
\text { competence }\end{array}$ & $\begin{array}{c}\text { Social } \\
\text { acceptance }\end{array}$ & $\begin{array}{l}\text { Athletic } \\
\text { competence }\end{array}$ & $\begin{array}{l}\text { Physical } \\
\text { appearance }\end{array}$ & $\begin{array}{l}\text { Behavioural } \\
\text { conduct }\end{array}$ & $\begin{array}{l}\text { Global } \\
\text { self-worth }\end{array}$ \\
\hline $\mathrm{R}$ squared & 0.319 & 0.295 & 0.165 & 0.365 & 0.424 & 0.540 \\
\hline $\begin{array}{l}\text { School } \\
\text { Age }\end{array}$ & 夫 & $\star$ & $\star \star$ & $\star \star$ & $\star \star$ & $\star \star$ \\
\hline $\begin{array}{l}\text { Father smokes } \\
\text { Mother smokes } \\
\text { Sister smokes } \\
\text { Brother smokes } \\
\text { Best friend smokes }\end{array}$ & $\begin{array}{l}\star \\
\star \\
\star \\
\star \\
\star\end{array}$ & $\begin{array}{c}\star \\
\star \\
\star \\
\star \\
0.238(0.073)\end{array}$ & $\begin{array}{l}\star \\
\star \\
\star \\
\star \\
\star\end{array}$ & $\begin{array}{c}\stackrel{\star}{\star} \\
\stackrel{\star}{\star} \\
0.222 \stackrel{(0.085)}{\star}\end{array}$ & $\begin{array}{l}\star \\
\star \\
\star \\
\star \\
\star\end{array}$ & $\begin{array}{l}\star \\
\star \\
\star \\
\star \\
\star\end{array}$ \\
\hline $\begin{array}{l}\text { Sometime smoker } \\
\text { Regular smoker }\end{array}$ & * & $\star$ & * & $\star$ & $\begin{array}{l}-0.686(0.219) \\
-0.391(0.098)\end{array}$ & $\star \star$ \\
\hline $\begin{array}{l}\text { Scholastic competence } \\
\text { Social acceptance } \\
\text { Physical appearance } \\
\text { Athletic competence } \\
\text { Behavioural conduct } \\
\text { Global self-worth }\end{array}$ & $\begin{array}{c}\stackrel{\star}{\star} \\
\star \\
0.092(0.038) \\
-0.252(0.230) \\
-0.243(0.195)\end{array}$ & $\begin{array}{c}\star \\
\star \\
\star \\
-0.537(0.162) \\
-0.887(0.165) \\
0.391(0.051)\end{array}$ & $\begin{array}{c}\star \\
-0.294(0.251) \\
-0.488(0.273) \\
\star \\
\star \\
\star\end{array}$ & $\begin{array}{c}\star \\
\star \\
\star \\
0.164(0.037) \\
\star \\
0.575(0.045)\end{array}$ & $\begin{array}{r}-0.404(0.204) \\
-0.094(0.046) \\
\star \\
-0.133(0.043) \\
\star \\
-0.196(0.176)\end{array}$ & $\begin{array}{r}-0.389(0.151) \\
0.217(0.036) \\
0.731(0.152) \\
\star \\
0.127(0.183) \\
\star\end{array}$ \\
\hline $\begin{array}{l}\text { Behavioural-global } \\
\text { Athletic-global } \\
\text { Social-physical } \\
\text { Scholastic-global } \\
\text { Social-athletic } \\
\text { Behavioural-scholastic } \\
\text { Physical-behavioural }\end{array}$ & $\begin{array}{c}0.195(0.071) \\
\star \\
\star \\
\star \\
\star \\
\star \\
\star\end{array}$ & $\begin{array}{c}\text { ^ } \\
0.270(0.055) \\
\star \\
\star \\
\star \\
\star \\
\star\end{array}$ & $\begin{array}{c}\star \\
\text { ^ } \\
0.227(0.087) \\
\star \\
\star \\
\star \\
\star\end{array}$ & $\begin{array}{l}\star \\
\star \\
\star \\
\star \\
\star \\
\star \\
\star\end{array}$ & $\begin{array}{c}\star \\
\star \\
\star \\
0.222(0.063) \\
0.152(0.073) \\
\star \\
\star\end{array}$ & $\begin{array}{c}\star \\
\star \\
\star \\
\star \\
\star \\
0.198(0.052) \\
-0.138(0.055)\end{array}$ \\
\hline Constant & $2.248(0.618)$ & $3.635(0.482)$ & $3.227(0.763)$ & $0.524(0.159)$ & $3.325(0.558)$ & $0.652(0.511)$ \\
\hline
\end{tabular}

Table 5(b) Girls: multiple linear regression models (parameter estimate, (SE)) with interactions with domains as the dependent variables $\left(^{\star}=N S\right.$

\begin{tabular}{|c|c|c|c|c|c|c|}
\hline & $\begin{array}{l}\text { Scholastic } \\
\text { competence }\end{array}$ & $\begin{array}{c}\text { Social } \\
\text { acceptance }\end{array}$ & $\begin{array}{l}\text { Athletic } \\
\text { competence }\end{array}$ & $\begin{array}{c}\text { Physical } \\
\text { appearance }\end{array}$ & $\begin{array}{l}\text { Behavioural } \\
\text { conduct }\end{array}$ & $\begin{array}{l}\text { Global } \\
\text { self-worth }\end{array}$ \\
\hline$R$ squared & 0.299 & 0.267 & 0.247 & 0.402 & 0.502 & 0.556 \\
\hline $\begin{array}{l}\text { School } \\
\text { Age }\end{array}$ & $0.111_{\star}^{(0.043)}$ & $\star \star$ & $\star \star$ & $\underset{\star}{0.102}(0.046)$ & $\begin{array}{l}0.379(0.205) \\
0.025(0.019)\end{array}$ & $-0.034^{\star}(0.014)$ \\
\hline $\begin{array}{l}\text { Father smokes } \\
\text { Mother smokes } \\
\text { Sister smokes } \\
\text { Brother smokes } \\
\text { Best friend smokes }\end{array}$ & $\begin{array}{l}\star \\
\star \\
\star \\
\star\end{array}$ & $\begin{array}{c}\star \\
\star \\
\star \\
\star \\
0.292(0.060)\end{array}$ & $\begin{array}{l}\star \\
\star \\
\star \\
\star \\
\star\end{array}$ & $\begin{array}{l}\star \\
\star \\
\star \\
\star \\
\star\end{array}$ & $\begin{array}{c}\star \\
\star \\
\star \\
\star \\
-0.249(0.064)\end{array}$ & $\begin{array}{l}\star \\
\star \\
\star \\
\star\end{array}$ \\
\hline $\begin{array}{l}\text { Sometime smoker } \\
\text { Regular smoker }\end{array}$ & $\star \star$ & $\star$ & $-0.206(0.097)$ & * & $\begin{array}{l}-0.698(0.543) \\
-0.458(0.088)\end{array}$ & $\star \star$ \\
\hline $\begin{array}{l}\text { Scholastic competence } \\
\text { Social acceptance } \\
\text { Physical appearance } \\
\text { Athletic competence } \\
\text { Behavioural conduct } \\
\text { Global self-worth }\end{array}$ & $\begin{array}{c}\star \\
\star \\
\star \\
\star \\
-0.056(0.179) \\
-0.049(0.171)\end{array}$ & $\begin{array}{c}\stackrel{\star}{\star} \\
\star \\
0.198 \stackrel{(0.036)}{\star} \\
0.360(0.047)\end{array}$ & $\begin{array}{c}\text { ^ } \\
0.383(0.055) \\
0.362(0.050) \\
\star \\
\star \\
\star\end{array}$ & $\begin{array}{c}{ }^{\star} \\
-0.097(0.048) \\
\star \\
0.193(0.037) \\
\star \\
0.609(0.049)\end{array}$ & $\begin{array}{c}-0.111(0.163) \\
\star \\
\star \\
\star \\
\star \\
0.068(0.153)\end{array}$ & $\begin{array}{c}0.178(0.040) \\
0.638(0.113) \\
0.823(0.148) \\
\star \\
0.217(0.040)\end{array}$ \\
\hline $\begin{array}{l}\text { Behavioural-global } \\
\text { Athletic-global } \\
\text { Social-physical } \\
\text { Scholastic-global } \\
\text { Social-athletic } \\
\text { Behavioural-scholastic } \\
\text { Physical-behavioural } \\
\text { Global-sometime } \\
\text { School-sometime } \\
\text { Age-sometime } \\
\text { Global-school }\end{array}$ & $\begin{array}{c}0.125(0.060) \\
\star \\
\star \\
\star \\
\star \\
\star \\
\star \\
\star \\
\star \\
\star\end{array}$ & $\begin{array}{l}\star \\
\star \\
\star \\
\star \\
\star \\
\star \\
\star \\
\star \\
\star \\
\star \\
\star\end{array}$ & $\begin{array}{l}\star \\
\star \\
\star \\
\star \\
\star \\
\star \\
\star \\
\star \\
\star \\
\star \\
\star\end{array}$ & $\begin{array}{l}\star \\
\star \\
\star \\
\star \\
\star \\
\star \\
\star \\
\star \\
\star \\
\star \\
\star\end{array}$ & $\begin{array}{c}\star \\
\star \\
\star \\
0.116(0.056) \\
\star \\
\star \\
\star \\
-0.275(0.083) \\
0.193(0.079) \\
0.080(0.036) \\
-0.177(0.070)\end{array}$ & $\begin{array}{c}\star \\
\star \\
-0.154(0.047) \\
\star \\
\star \\
\star \\
\star \\
\star \\
\star \\
\star \\
\star\end{array}$ \\
\hline Constant & $1.889(0.489)$ & $1.415(0.134)$ & $0.543(0.181)$ & $0.335(0.151)$ & $1.937(0.488)$ & $-0.539(0.417)$ \\
\hline
\end{tabular}

domains, and smoking status, two regression analyses were carried out. The first, described in this section, was a multiple linear regression analysis, with each domain taken in turn as the dependent variable. The independent variables included in the modelling were as follows: school attended, age, father smokes, mother smokes, brother smokes, sister smokes, best friend smokes, and the self-perception domains. Main affects were modelled and firstorder significant main affect interactions were investigated. The findings are shown in table 5.

Perhaps surprisingly in view of the odds ratios described above, no aspect of smoking was significant in the models for scholastic competence or global self-worth for either boys or girls. However, one point of interest here was the inclusion of school as a main affect factor for girls but not for boys with regard to self-perception of their own scholastic competence.

Likewise no aspect of smoking featured significantly in the model related to selfperceived physical appearance for girls, whilst for boys, having a brother who smoked was a main affect.

When athletic competence was taken as the dependent variable, regular smoking was negatively related to high athletic self-perception for girls, but did not appear in the model for boys. 
Table 6 Logistic regression models taking current smoker/not current smoker as dependent variables (by gender)

\begin{tabular}{|c|c|c|c|c|c|c|}
\hline \multirow[b]{2}{*}{ Significant variables } & \multicolumn{3}{|c|}{ Boys } & \multicolumn{3}{|c|}{ Girls } \\
\hline & $B$ & $(S E)$ & $p$ value & $B$ & $(S E)$ & $p$ value \\
\hline $\begin{array}{l}\text { Best friend smokes } \\
\text { Father smokes }\end{array}$ & $\begin{array}{l}3.741 \\
2.102\end{array}$ & $\begin{array}{l}(0.540) \\
(0.562)\end{array}$ & $\begin{array}{l}<0.001 \\
<0.001\end{array}$ & 3.267 & $(0.426)$ & $<0.001$ \\
\hline $\begin{array}{l}\text { Age } 15 \\
\text { Brother smokes }\end{array}$ & 1.496 & $(0.607)$ & 0.014 & 1.474 & $(0.449)$ & 0.001 \\
\hline $\begin{array}{l}\text { Social acceptance } \\
\text { Behavioural conduct } \\
\text { Mother smokes }\end{array}$ & $\begin{array}{l}-1.193 \\
-1.787\end{array}$ & $\begin{array}{l}(0.460) \\
(0.690)\end{array}$ & $\begin{array}{r}<0.001 \\
0.010\end{array}$ & $\begin{array}{r}0.955 \\
-1.647\end{array}$ & $\begin{array}{l}(0.457) \\
(0.463)\end{array}$ & $\begin{array}{r}0.037 \\
<0.001\end{array}$ \\
\hline $\begin{array}{l}\text { School attended } \\
\text { Constant }\end{array}$ & -1.125 & $(1.228)$ & 0.360 & $\begin{array}{l}-0.958 \\
-1.682\end{array}$ & $\begin{array}{l}(0.451) \\
(1.724)\end{array}$ & $\begin{array}{l}0.034 \\
0.329\end{array}$ \\
\hline
\end{tabular}

Most interesting was the appearance of "best friend smokes" as a main affect in relation to a high social acceptance score for both boys and girls. But it was when perception of their own behavioural conduct was the dependent variable that the most complex model with regard to smoking was obtained.

LOGISTIC REGRESSION MODELLING OF FACTORS ASSOCIATED WITH CURRENT SMOKING OR NOT SMOKING

As described in the methodology section, the following variables were included in the analysis: school attended, age, father smokes, mother smokes, brother smokes, sister smokes, best friend smokes, social acceptance, behavioural conduct, physical appearance, scholastic competence, athletic competence, and global selfworth. The model for the boys correctly predicted $94 \%$ of the overall outcome, $98 \%$ of current non-smokers and $45 \%$ of current smokers, with five boys having standardised residuals higher than 3 . For girls, the model correctly predicted $92 \%$ of the overall smokers, $96 \%$ of current non-smokers and $71 \%$ of current smokers, with six girls having standardised residuals higher than 3 .

As table 6 shows, best friend's smoking and lower behavioural conduct perception scores were the most significant factors in the model associated with current smoking for both boys and girls. For girls, being aged 15, having a higher social acceptance score, and attending one rather than the other of the schools were also significantly associated with current smoking.

For boys, a low behavioural conduct score was important in the model, with smoking among other male members of the family (including both father and brother) being significant. A rather strange artifact was produced which caused mother's smoking to appear to reduce the risk of smoking in boys. However, it was observed that the mothers were more likely to be smokers if the father also smoked ( $\mathrm{OR}=5.59 ; 95 \% \mathrm{CI}=3.35-$ 9.33). Consequently, very few of the boys lived in a household where only the mother smoked.

\section{Discussion}

It is important that the findings of a relatively small, cross-sectional survey of the kind described in this paper are not over-interpreted. For example, causality cannot be implied from the associations. What the study does provide is an insight into self-perception measured by a validated psychological instrument which enables the various elements of self-image to be separately assessed and their interrelationships to be determined. In this way, their association with being a smoker or a nonsmoker at a single point in time can be examined.

Numerous studies have considered the involvement of self-perception or self-esteem in smoking, but most have taken an overall view, rather than focusing on specific domains. ${ }^{11,12}$ The only study the authors know in Britain which has used Harter's Self-Perception Profile for Children in relation to smoking did find lower self-esteem in girls aged 11 to 13 than in boys of the same age, ${ }^{10}$ a fall in girls' selfperception with increasing age, and an association between low self-esteem and smoking, especially in girls. Therefore the findings described in this study corroborate these findings and take the analysis further.

Some studies have considered male students and their intentions to smoke, related to their level of self-esteem, ${ }^{13,14}$ whilst others have focused on females. ${ }^{15}$ Few appear to have related smoking to the domains of physical appearance or to the generally lower selfperception among girls than among boys by using a validated psychological test. In 1986, Penny and Robinson ${ }^{16}$ suggested that smoking was used by adolescents with a low sense of personal effectiveness as a means of stress management. Self-esteem has also been seen in the light of respect for one's own body, in which case an approach to increasing selfesteem was seen as being increasing the young person's health knowledge. ${ }^{17}$ Lack of selfesteem as translated into a lack of skills to refuse cigarettes has also been an interpretation of the message. ${ }^{18}$ However, from our study it seems more likely to be a lack of desire to refuse rather than a lack of skills, which could account for the relative lack of success of some social skills-based teaching programmes.

The fact that girls' self-perception, especially with regard to physical appearance and global self-worth, was so much lower than that of boys and that girls' self-perception scores decreased steadily in successive cohorts from bottom to top of the age range suggests, smoking education apart, that there is a need to look more deeply into this phenomenon. The significance of the school attended, in relation to scholastic, physical, and behavioural selfperception for girls, but not for boys, suggests that external factors as well as developmental ones are influential. A health-promoting school is responsible for much more than prevention of smoking. The well-being and self-image of the students is also part of its responsibility. Achieving their maximum potential and being happy in this achievement is a right of every student. Scholastic competence is by no means the only way in which a student can achieve. Perhaps the first thing to be achieved by a health-promoting school is an ethos with realistic and attainable goals, with due acknowledgement when these are reached. 
Physical appearance is of prime importance to adolescent girls as all magazines targeted at this age group appear to insist it should be. However, these publications feature and applaud young women who epitomise the current image of beauty and attractiveness. At the age of 12 to 15 years, and perhaps always, this image is completely unattainable for most girls. Consequently they are dissatisfied with their hair, weight, shape, and skin and, because they do not fit the stylised image, they are unhappy about their appearance. The study showed that physical appearance and global self-worth were very significantly associated for girls, but not for boys. It was the largest significant link found between domains. Schools usually spend little time on physical appearance. Helping girls to make the best of themselves, to make changes where necessary and possible, but above all to help them to accept themselves as they are, are most important roles for a healthpromoting school.

Many young people reject "adult" values ${ }^{19}$ and the low self-perception among smokers with regard to behavioural and scholastic competence could be a reflection of this rejection. Many studies have shown increased risk of smoking among rebellious young people and among underachievers. The question arises as to how the health-promoting school can approach this rejection of adult norms. One suggestion is that it should be accepted as an integral part of adolescence and, rather than being fought, it should be harnessed or channelled towards positive ends which are of value to the young person. For example, many rebellious adolescents have a strong sense of justice. They will often see the injustice of, for example, tobacco marketing in developing countries, and be spurred into action as few adults might be.

All models of health education or health promotion, apart from Knowledge, Attitudes, and Practices (KAP), have included a personal element related to self-perception. Fishbein and Ajzen implicitly include it in both the attitudinal and the normative beliefs of the Theory of Reasoned Action." "What will smoking do for me and how will other people view me if I smoke?" The high social acceptance scores of the smokers suggests that both these elements are in play, especially in view of a best friend smoking being a main effect in association with this domain. The health-promoting school is in the business of helping all students with their social acceptance among their peers without smoking forming a necessary part. Social skills extend far beyond refusal of cigarettes. No skills training in the world will stop a young person accepting a cigarette if they do not want to say "No thank you". Friendships, personal relationships, and having fun are essential elements in the health-promoting school's curriculum, either taught or hidden.

The logistic regression analysis showed that self-perception domains were not the most significant factors related to smoking in these young people. Friends' smoking, and to some extent family smoking, were more important.
However, behaviour with regard to friends is related to self-perception. Tones's Health Belief Model $^{21}$ and his related Drug Use Model $^{22}$ both show how the family and friends influence self-esteem, which in turn influences decisions on health behaviour.

As a result of the findings of this pilot study, the authors have now embarked on a longitudinal study of smoking, self-perception, and other background factors from the age of 9 years (Year 5) to 14 (Year 9). In this way we hope to monitor changes and perhaps to identify causative links. Cluster analysis will be possible. We hope this longitudinal study will help to pinpoint stages and changes which will provide keys to planning and targeting health promotion.

The authors sincerely thank the Cancer Research Campaign and the Japanese Ministry of Education for their financial support; Dr Susan Harter of Denver, Colorado for permission to modify and use her Self-Perception Scale; the Directors of Education for their permission to carry out the study; the headteachers, staff, and pupils of the two schools without whose help this study would not have been possible.

1 Office of Population Censuses and Surveys. General Household Survey: Cigarette Smoking 1972-1990 OPCS Monitor SS91/3. London: OPCS, 1991.

2 Lader D, Matheson J. Smoking among Secondary School Children in 1990. An enquiry carried out by the Social Survey Division of OPCS on behalf of the Department of Health, the Welsh Office and the Scottish Office Home and Health Department. London: HMSO, 1991.

3 Charlton A, Melia P, Moyer CA (eds). A Manual on Tobacco and Young People for the Industrialised World. Tobacco and Young People for the Industrialised World.

4 Royal College of Physicians. Smoking and the Young: A Report of a Working Party of the Royal College of Physicians. London: Royal College of Physicians, 1992.

5 Marsh A, Matheson J. Smoking Attitudes and Behaviour. London, HMSO, 1983.

$6 \mathrm{McNeill}$ AD. The development of dependence on smoking in children. $B r \mathcal{F}$ Addict 1991; 86: 589-92.

7 Department of Education and Science. Education Statistics for the United Kingdom 1991 Edition. Government Statistical Service Publication. London: HMSO, 1992.

8 Bewley BR, Day I, Ide L. Smoking by Children in Great Britain. A Review of the Literature. London: Social Science Research Council/Medical Resource Council, 1972.

9 Harter S. Manual for the Self-Perception Profile for Children. Denver: University of Denver, 1985.

10 Lloyd P. Self-esteem and smoking in 11 to 13-year-olds. MSc Thesis. Manchester: University of Manchester, 1989.

11 Newcombe MD, Maddahian E, Bentler PM. Risk factors for drug use among adolescents: concurrent and longitudinal analysis. Am $\mathcal{f}$ Public Health 1986; 76: 525-31.

12 Allendorff S, Sunseri AJ, Cullinan J et al. Student heart health knowledge, smoking attitudes, and self-esteem. f Sch Health 1985; 55: 196-9.

13 Tucker LA. Psychological differences between adolescent smoking intenders and nonintenders. F Psychol 1984; 118: $37-43$.

14 Tucker LA. Physical, psychological, social and lifestyle differences among adolescents classified according to differences among adolescents classified according to
cigarette smoking intention status. $\mathcal{F}$ Sch Health 1985; 55: 127-31.

15 Tax HS. Self-esteem, participation in extracurricular activities and cigarette smoking in adolescent females. Doctoral dissertation. New York: New York University. University Microfilms International 83-13878 (112 pp) 1983.

16 Penny GN, Robinson JO. Psychological resources and cigarette smoking in adolescents. Br $\mathcal{F}$ Psychol 1986; 77 : $351-7$

17 Wynder EL. The etiology, epidemiology and prevention of lung cancer. Semin Respir Med 1982; 3: 135-9.

18 Perry CL, Murray DM. Enhancing the transition years: the challenge of adolescent health promotion. $\mathcal{F}$ Sch Health 1982; 52: 307-11.

19 Jessor R. Problem behaviour and developmental transition in adolescence. $\mathcal{F}$ Sch Health 1982; 23: 189-99.

20 Fishbein M, Ajzen J. Belief, Attitude, Intention and Behaviour: an Introduction to Theory and Research. Rehaviour: an Introduction to Theory and Resec

21 Tones BK. Health Promotion, affective education and the personal-social development and young people. In: Health Education in Schools, Second Edition. $\mathrm{K}$ David, $\mathrm{T}$ Williams, eds. London: Harper and Row, 1987.

22 Tones K, Tilford S, Robinson Y. Health Education: Effectiveness and Efficiency. London: Chapman Hall, 1990. 